\title{
Desejabilidade Social e Precisão do Inventário de Habilidades Sociais para Cuidadores de Idosos (IHS-CI)
}

\section{Social Desirability and Reliability of the Social Skills Inventory for Caregivers of Elderly People (IHS-CI)}

\author{
Francine Náthalie Ferraresi Rodrigues Queluz ${ }^{1}$, Acácia Aparecida Angeli dos Santos ${ }^{2}$ \\ e Luziane de Fátima Kirchner ${ }^{3}$
}

\begin{abstract}
Resumo
O Inventário de Habilidades Sociais para Cuidadores de Idosos (IHS-CI) foi desenvolvido para avaliar o repertório interpessoal de cuidadores familiares. $\mathrm{O}$ instrumento apresenta evidências de validade interna e externa. No entanto, questiona-se a existência do viés desejabilidade social. O objetivo deste estudo foi verificar as evidências de validade discriminativa em relação à desejabilidade social e precisão do IHS-CI. Participaram 252 cuidadores de idosos, idade média de 50 anos $(D P=14.4)$, que responderam aos seguintes instrumentos: Questionário Sociodemográfico, Critério Brasil, IHS-CI e Escala de Desejabilidade Social Marlowe-Crowne. As habilidades sociais não se correlacionaram com a desejabilidade social, o que indica que o IHS-CI apresenta evidências de validade discriminativa. Os valores de precisão encontrados foram: Expressividade Afetiva ( $\omega=.85)$; Comunicação Assertiva $(\omega=.77)$; Busca de Formação/Informação $(\omega=.68)$ e Escore Geral $(\omega=.90)$. O IHS-CI está apto para uso e apresenta boas evidências de validade.
\end{abstract}

Palavras-chave: competência social, assertividade, envelhecimento, avaliação psicológica, psicometria

\begin{abstract}
The Social Skills Inventory for Caregivers of the Elderly People (SSI-CE) was developed to assess the interpersonal repertoire of family caregivers. The instrument presents evidence of internal and external validity. However, the existence of a social desirability bias is questioned. The aim of this study was to verify the evidence of discriminative validity in relation to the social desirability and the reliability of the IHS-CI. The sample comprised 252 caregivers of elderly people, mean age of 50 years-old $(\mathrm{SD}=14.4)$, who answered the following instruments: Sociodemographic Questionnaire; Brazil Criteria; IHS-CI; and Marlowe-Crowne Social Desirability Scale. Social skills did not correlate with social desirability, which indicates that the IHS-CI presents evidence of discriminative validity. The precision values found were: Affective Expressiveness $(\omega=.85)$; Assertive Communication $(\omega=.77)$; Training/Information Search $(\omega=.68)$ and General Score $(\omega=.90)$. The IHS-CI is adequate for use and has strong evidence of validity.
\end{abstract}

Keywords: social competence, assertiveness, ageing, psychological assessment, psychometrics

\footnotetext{
${ }^{1}$ Mestre e Doutora em Psicologia, Pós-doutoranda em Psicologia na Universidade São Francisco. Rua Waldemar Cesar da Silveira, 105, Jardim Cura D'ars, Campinas - SP, Brasil. Tel.: +55 19995416828. E-mail: francine.queluz@gmail.com

${ }_{2}^{2}$ Psicóloga, Mestre e Doutora em Psicologia, Professora Titular da Universidade São Francisco. Rua Waldemar Cesar da Silveira, 105, Jardim Cura D’ars, Campinas - SP, Brasil. Tel.: +55 19981418899. E-mail: acacia.angeli@gmail.com

${ }^{3}$ Psicóloga, Mestre em Análise do Comportamento, Doutora em Psicologia, Professora da Universidade Católica Dom Bosco. Universidade Católica Dom Bosco. Programa de Pós-Graduação em Psicologia. Avenida Tamandaré, 6000, Jardim Seminário, Campo Grande-MS, Brasil. Tel.: +55 6781902100. E-mail: luzianefk@ucdb.br
} 


\section{Introdução}

Cuidar de um familiar idoso tem sido considerado algo normativo, o qual todos precisarão fazer em algum momento da vida (Keating, Eales, Funk, Fast, \& Min, 2019). Essa demanda tem sido alta, especialmente ao se levar em consideração o aumento atual na quantidade de idosos, em comparação à década anterior (United Nations, 2019). No entanto, assumir a tarefa de cuidar de uma pessoa dependente pode ter um impacto negativo, como o aumento do estresse, da sobrecarga, de conflitos com pessoas envolvidas no contexto, além de uma maior probabilidade de surgimento de sintomas depressivos (Wank, Robinson, \& Carter-Harris, 2014). Esses prejuízos podem ser reduzidos mediante a presença de um bom repertório de habilidades sociais, o qual atua como fator protetivo ao indivíduo (Del Prette \& Del Prette, 2019). Segundo Del Prette e Del Prette (2019), as habilidades sociais são desenvolvidas ao longo da vida e contribuem para melhorar a qualidade dos relacionamentos interpessoais de maneira geral. Estudos voltados à avaliação deste repertório em cuidadores de idosos são escassos e, para realizálos, se faz necessário aprimorar a qualidade dos instrumentos desenvolvidos.

Queluz, Barham, Del Prette, Fontaine e Olaz (2017) desenvolveram um instrumento para avaliar as habilidades sociais de cuidadores de idosos, o qual foi testado em relação a evidências de validade interna e externa. Dando continuidade à análise psicométrica deste instrumento, o presente estudo objetivou avaliar as propriedades psicométricas do Inventário de Habilidades Sociais para Cuidadores de Idosos (IHS-CI), no que diz respeito à precisão e à presença de desejabilidade social.

Os cuidadores normalmente são familiares, do sexo feminino (majoritariamente filhas ou esposas), com idade entre 50 e 65 anos que moram relativamente perto ou na mesma residência que o idoso cuidado (Vlachantoni, \& Palmer, 2019; Wank et al., 2014). De acordo com $\mathrm{Fu}$ (2019), as demandas do idoso, inerentes ao cuidado, podem ser físicas ou cognitivas ou, ainda, envolver as duas concomitantemente. As demandas físicas referem-se às restrições de movimentos ou capacidades sensoriais, que fazem com que o idoso possa precisar de ajuda para comer, higienizar-se, deslocar-se dentro e fora de sua residência, entre outros. As demandas cognitivas dizem respeito aos idosos com transtornos neurocognitivos, que não conseguem se lembrar de informações importantes, se comunicar adequadamente ou realizar tarefas baseadas no raciocínio (Fu, 2019). Devido ao comprometimento cumulativo da memória, com o passar do tempo, o idoso que apresenta transtorno neurocognitivo passa a não se recordar de como realizar as tarefas diárias, o que acarreta dificuldades na condição física também. Com o tempo, as dificuldades do cuidador para se adaptar e para lidar com essas demandas, podem levá-lo ao desenvolvimento de estresse e sentimentos de sobrecarga (Fu, 2019; Zarit \& Polenick, 2019).

De acordo com Del Prette e Del Prette (2019), a presença de um repertório de habilidades sociais bem desenvolvido pode ser um fator protetivo para lidar com adversidades em diferentes contextos de vida. Ainda de acordo com esses autores, as habilidades sociais são definidas como um construto descritivo de comportamentos sociais valorizados em determinada cultura que colaboram para que os relacionamentos interpessoais sejam de qualidade. Especificamente no contexto da assistência prestada a um familiar dependente, elas podem colaborar para a manutenção de relacionamentos de qualidade com toda a família, o que consequentemente fortalece o apoio mútuo, e faz com que o cuidador sinta um menor impacto na sua saúde mental ao exercer a tarefa de cuidar de seu familiar (Queluz, Barham, \& Del Prette, 2019).

As classes de habilidades sociais são comportamentos que podem ser aprendidos ao longo da vida, como por exemplo, expressar sentimentos positivos, comportar-se assertivamente ou empaticamente, controlar a agressividade, estabelecer e manter conversação, apresentar comportamentos de civilidade. Todos estes podem colaborar para o desenvolvimento e a manutenção de relacionamentos interpessoais saudáveis (Del Prette \& Del Prette, 2019; Pinto, Barham, \& Del Prette, 2016; Morán, García, \& Hormazabal, 2018; Salas, Asún, \& Zúñiga, 2020). Cabe também ressaltar que as habilidades sociais requeridas são diferentes, a depender de cada contexto. Por exemplo, no ensino superior, é 
esperado que os alunos consigam ter a habilidade de se apresentar em público, já no contexto do cuidado, a habilidade de controlar a agressividade frente a uma situação adversa com o idoso poderá ter maior importância, principalmente se o idoso tiver algum tipo de demência.

Apesar da relevância deste campo de investigação, ainda é escassa a quantidade de estudos que visam avaliar as habilidades sociais de pessoas que prestam assistência a um familiar (Queluz et al., 2019) e, mais escassa ainda, a quantidade de estudos que desenvolveram $\mathrm{e}$ avaliaram instrumentos específicos para a avaliação das habilidades sociais de cuidadores de idosos (Queluz et al. 2017; Queluz, Barham, Del Prette, \& Santos, 2018).

Alguns destes estudos são descritos em seguida. Por exemplo, Pinto et al. (2016) realizaram um estudo com 50 cuidadores de idosos familiares, 25 idosos lúcidos cuidados e 25 profissionais da saúde que trabalhavam com envelhecimento, para identificar quais habilidades sociais seriam mais requeridas no contexto de prestar assistência a outrem. As habilidades mais relatadas, de maneira concomitante para todos esses grupos, foram: obter informação acerca da doença, expressar sentimentos positivos, controlar a agressividade, conversar para resolver problemas e solicitar ajuda, quando necessário. As autoras do estudo concluíram, com base nos dados encontrados, que um cuidador com habilidades sociais bem desenvolvidas consegue conciliar as suas próprias necessidades com as do idoso cuidado e de outros familiares envolvidos, sempre tentando manter as relações interpessoais saudáveis e positivas. Exemplos disto são agradecer quando receber ajuda de outras pessoas ou expressar sentimentos positivos ao idoso de diferentes formas (falando, fazendo uma comida ou atividade que ambos gostam).

Mais recentemente, Queluz et al. (2019) realizaram uma revisão da literatura de estudos que avaliaram ou que realizaram intervenções voltadas ao desenvolvimento das habilidades sociais em cuidadores de idosos. Foram encontrados onze estudos: cinco apresentaram intervenções, quatro apresentaram análises correlacionais, um apresentou análise qualitativa e um descreveu a elaboração de um instrumento de competência social. As buscas foram realizadas sem limitar uma data de início até o final de 2016, tendo uma diferença de três anos entre a publicação e o levantamento dos dados. Notou-se que os autores dos estudos interviram, mas não avaliaram as habilidades sociais de cuidadores, ou utilizaram os instrumentos com evidências de validade obtidas junto a outras populações, o que desconsidera a característica situacional das habilidades sociais da população pesquisada. $\mathrm{O}$ estudo que apresentou a elaboração do instrumento de competência social teve somente cuidadores formais pagos como participantes, desconsiderando os cuidadores familiares que normalmente são a maioria e que não podem abandonar a tarefa, caso tenham sua saúde impactada. Ademais, nos estudos correlacionais, as variáveis relacionadas ao bom repertório de habilidades sociais de cuidadores foram: menor percepção de sobrecarga e estresse, menor presença de sintomas depressivos e de conflitos com o idoso cuidado, além de uma maior autoestima, maior presença de suporte social e melhor percepção de qualidade de vida. Tendo em vista os resultados positivos, relacionando as habilidades sociais a todas essas variáveis, as autoras da revisão concluíram que as avaliações poderiam apresentar resultados mais robustos ou subsidiar de maneira mais efetiva as intervenções, se tivessem sido realizadas com um instrumento, especificamente construído para avaliar as habilidades sociais de cuidadores familiares de idosos.

Considerando a inexistência deste instrumento, Queluz et al. (2017) desenvolveram o Inventário de Habilidades Sociais para Cuidadores de Idosos familiares. Os autores apresentaram inicialmente evidências de validade baseadas no conteúdo, analisando os aspectos semânticos e de pertinência teórica para a primeira versão do IHS-CI. Sequencialmente, apresentaram as evidências de validade baseadas na estrutura interna por meio de uma análise fatorial exploratória, com medidas de precisão adequadas, tal como identificada por meio do Alpha de Cronbach. Posterior a esta análise, foi encontrada uma estrutura com 24 itens, divididos em três fatores: "Expressividade afetiva" (10 itens, $\alpha=.87)$; "Comunicação assertiva" (10 itens, $\alpha=.79)$; "Busca por Formação/Informação $(\alpha=.60)$, além do Escore Total $(\alpha=.89)$. Quanto 
maior a pontuação do cuidador, maior o seu repertório interpessoal. Segundo Queluz et al. (2017), a Expressividade Afetiva está relacionada à expressão de sentimentos positivos para todas as pessoas envolvidas no cuidado, enquanto a Comunicação Assertiva se refere ao exercício de direitos em relação ao cuidador e ao idoso e ao enfrentamento de situações indesejadas, mesmo que com risco de contrariar o outro. Por sua vez, a Busca por Formação/Informação é a habilidade do cuidador de angariar informações para melhorar a assistência ao seu parente idoso, isto pode ser feito ao tirar dúvidas com outros cuidadores ou com profissionais da área de saúde.

Em um estudo posterior, Queluz et al. (2018) buscaram evidências de validade baseadas na relação com variáveis externas, por meio da verificação da relação das medidas do IHS-CI com os construtos depressão, sobrecarga, qualidade de vida e qualidade da relação diádica cuidador-idoso. Participaram do estudo 205 cuidadores familiares de idosos, que responderam ao IHS-CI, a Escala de Relacionamento da Díade, ao Inventário de Sobrecarga de Zarit (Scazufca, 2002), ao Inventário de Depressão de Beck (Beck, Rush, Shaw, \& Emery, 1979) e à Escala de Qualidade de vida de Novelli (Novelli, 2006). Como resultado, o maior escore de habilidades sociais apresentado pelos cuidadores se correlacionou positivamente com qualidade de vida e qualidade da relação diádica, e negativamente com presença de sintomas depressivos, sobrecarga e presença de conflitos na díade, indicando evidências de validade baseada na relação com variáveis externas para o instrumento.

Mais recentemente, Queluz, Santos, Ximenes, Batista e Kircher (2020), realizaram um outro estudo com o IHS-CI buscando verificar se a estrutura fatorial encontrada por Queluz et al. (2017) se manteria (evidências de validade interna), quais seriam os valores de precisão em uma nova amostra e se o instrumento de relacionaria com a empatia (novas evidências de validade baseada na relação com variáveis externas), uma vez que esta é uma classe de habilidades sociais. Participaram 252 cuidadores de idosos familiares, que responderam ao IHS-CI e a um Questionário sociodemográfico, e dentre estes, 74 responderam também ao Inventário de
Empatia. Após a realização da análise fatorial confirmatória, a estrutura fatorial do IHS-CI mostrou-se adequada (RMSEA $=0.07 ; \mathrm{CFI}=0.92 \mathrm{e}$ $\mathrm{x}^{2} / \mathrm{gl}=2.35$ ) e se correlacionou positivamente com as medidas de empatia. Além disto, os valores de precisão foram considerados aceitáveis (Fator Expressividade Afetiva, $\alpha=.85$; $\quad$ Fator Comunicação Assertiva, $\alpha=.73$; Fator Busca de Formação/Informação, $\alpha=.68$ e Escore Geral, $\alpha=.89$ ).

Dado o exposto, percebe-se que as propriedades psicométricas do IHS-CI são adequadas e indicam, de forma promissora, que a teoria está bem representada pelo instrumento. Isto é fundamental, uma vez que permite seguir com investigações em relação às suas evidências de validade. Uma característica que tem sido muito investigada no processo de busca de evidências de validade, é se o instrumento apresenta algum tipo de viés de resposta (Almiro, 2017), pois isso indicaria que os participantes não estão respondendo aos itens de forma coerente ao que seria esperado para o construto que eles pretendem medir. Segundo Almiro (2017), um dos principais vieses de resposta que pode atrapalhar na medida do construto é a desejabilidade social.

Segundo Marlowe e Crowne (1961), a desejabilidade social pode ser definida como a probabilidade de os participantes responderem aos itens de um instrumento atribuindo a si mesmo, de forma intencional ou não, respostas que seriam desejáveis dentro do contexto em que se inserem. Isso pode ser ainda mais preocupante ao considerar um instrumento que visa medir as habilidades sociais, uma vez que tais habilidades são desejáveis para o convívio social (Del Prette \& Del Prette, 2019). Diante disto, é importante que o instrumento consiga aferir o que se propõe, e tenha o mínimo de influência possível deste tipo de viés de resposta (Almiro, 2017).

Ademais, outra característica a se atentar no processo de construção é se um instrumento apresenta bons índices de precisão. Conforme já exposto, o IHS-CI apresentou índices aceitáveis ao se considerar os valores do Alpha de Cronbach obtidos em estudos anteriores, tanto para o escore geral como para os fatores (Queluz et al., 2017; Queluz et al. 2020). Segundo Ventura-León e Caycho-Rodríguez (2017), o Alpha de Cronbach, apesar de ser a medida mais utilizada para avaliar 
a precisão em ciências humanas, apresenta algumas limitações. Por exemplo, ele pode ser afetado pelo número de itens e pelo número de alternativas de respostas, sendo a sua vantagem a possibilidade do cálculo com uma única aplicação do instrumento. Diante dessas limitações, os autores sugeriram o uso do coeficiente ômega de McDonald para medir a precisão, uma vez que este considera as cargas fatoriais padronizadas, fazendo com que os cálculos sejam mais estáveis, o que por sua vez, faz com que as medidas reflitam um valor de precisão mais próximo ao verdadeiro.

Conclui-se, então, que quanto mais evidências de validade um instrumento apresentar, maiores serão os indícios de que ele mede o que se propõe e de que está apto para uso (AERA, APA, \& NMCE, 2014). A partir destas considerações, o objetivo deste estudo foi verificar as evidências de validade discriminativa em relação à desejabilidade social e a precisão do IHS-CI por meio do ômega de McDonald. Considerando que desejabilidade social é um viés de resposta (Almiro, 2017), partiu-se da hipótese de que o IHS-CI não apresentaria correlação com este tipo de viés e as medidas de precisão estariam dentro dos níveis aceitáveis (Queluz et al., 2017; Queluz et al., 2020).

\section{Método}

\section{Participantes}

Participaram deste estudo 252 cuidadores de idosos familiares, com idade média de 50 anos $(\mathrm{DP}=14.4)$, variando entre 18 e 79 anos, sendo $219(86.9 \%)$ do sexo feminino e $33(13.1 \%)$ do sexo masculino. A maioria era filho $(60.7 \%)$, cônjuge (13.9\%) ou neto do idoso (9.5\%), seguido por nora ou genro $(7.9 \%)$, outros $(6.8 \%)$, ou irmãos $(1.2 \%)$. No que diz respeito à categoria outros, estes poderiam ser sobrinhos, vizinhos, amigos, opções que não constavam no questionário sociodemográfico. Em relação ao estado civil, a maioria era casado (67.9\%), seguido de solteiros (19.8\%), separados $(9.1 \%)$ e viúvos (3.2\%). Na amostra coletada, eram todos brasileiros, sendo que predominou a quantidade de residentes no estado de São Paulo (69.4\%), seguido de Rio de Janeiro e Rondônia (9.9\% cada), Paraná (6.0\%) e Amazonas (4.8\%). A maior parte dos cuidadores apresentou Ensino Superior completo (40.9\%), seguido de Ensino Médio completo $(36.5 \%)$, até $4^{\mathrm{a}}$ série $(11.1 \%)$, Ensino Fundamental completo (6.3\%) e Analfabeto ou até a $3^{\mathrm{a}}$ série $(5.2 \%)$. A análise do Critério Brasil (Associação Brasileira de Empresas e Pesquisa [ABEP], 2016) mostrou que a maioria dos cuidadores pertencia ao grupo B2 (31.0\%), seguido de B1 (21.4\%), C1 (19.0\%), A (13.5\%), C2 (9.5\%), D (4.4\%) e E (1.2\%). A amostragem foi não probabilística, por conveniência.

Os cuidadores foram recrutados junto ao programa nacional brasileiro denominado Serviço de Atendimento Domiciliar (SAD) em cada estado ou via indicação de outros cuidadores ou conhecidos dos pesquisadores. Como critério de inclusão, os participantes teriam que ser maiores de 18 anos, e familiares dos idosos que cuidavam. Caso os cuidadores recebessem algum pagamento para cuidar do idoso, eles eram excluídos da amostra. A coleta de dados foi realizada nas residências dos cuidadores, nos $\mathrm{SAD}$ ou em algum lugar escolhido por eles, de forma a garantir sigilo e privacidade.

\section{Instrumentos}

\section{Questionário sociodemográfico}

Um questionário foi elaborado para levantar as características sociodemográficas dos cuidadores, sendo elas: sexo, idade, escolaridade, estado civil e relação com o idoso cuidado.

\section{Critério de Classificação Econômica Brasil (ABEP, 2016)}

Este instrumento avalia o nível socioeconômico das famílias com base no poder aquisitivo, no número de bens de consumo duráveis, na presença de empregada doméstica e no grau de instrução do chefe da família, dividindo a população em sete classes (A, B1, B2, C1, C2, D e E).

\section{Inventário de Habilidades Sociais para Cuidadores de Idosos Familiares - IHS- CI (Queluz et al., 2017) \\ O IHS-CI é constituído por 24 itens, divididos em três fatores: "Expressividade afetiva" (10 itens, $\alpha=.87$ ), "Comunicação assertiva" (10 itens,}


$\alpha=.79)$ e "Busca por Formação/Informação (4 itens, $\alpha=.60)$ e apresenta alfa de Cronbach total de .89. O primeiro e o segundo fator geram um escore que pode variar entre 10 a 40 e o terceiro gera um escore que pode variar entre 4 e 12. A soma dos fatores resulta em um escore geral que pode variar de 24 a 96 . Quanto maior o valor, maior o repertório de habilidades sociais relatado pelo cuidador.

\section{Marlowe-Crowne Social Desirability Scale,} versão brasileira de Gouveia et al. (2009).

É um instrumento de autorrelato destinado a avaliar a desejabilidade social. Esta escala é composta por 20 itens que descrevem comportamentos aceitáveis, mas improváveis (itens de atribuição), assim como comportamentos inaceitáveis, mas prováveis (itens de negação). Para responder a esta escala, o indivíduo deve indicar se concorda ou não com o item (no caso, verdadeiro ou falso). As pontuações obtidas podem variar entre 0 e 20 sendo que quanto maior a pontuação, maior será a desejabilidade social presente nas respostas do participante (Crowne \& Marlowe, 1960). Os estudos para avaliar as evidências de validade desta escala no Brasil demonstram que o instrumento possui boa consistência interna de .75 (Gouveia et al., 2009).

\section{Procedimento}

A parte inicial do estudo consistiu em obter autorização das instituições para coleta de dados e em seguida, a submissão e aprovação pelo Comitê de Ética da Universidade São Francisco (Parecer $\mathrm{N}^{\circ}$ 1.876.754/2017). Após a aprovação, houve um novo contato com as instituições para selecionar os cuidadores com o perfil desejado. Feito o contato com os cuidadores e convidando-os a participar do estudo, foi combinado um lugar para a coleta dos dados, que garantisse o sigilo e a privacidade dos participantes. Antes da coleta, foi apresentado verbalmente e por escrito o Termo de Consentimento Livre e Esclarecido (TCLE), e sanadas as dúvidas remanescentes a pesquisa. Em seguida, os cuidadores que concordaram em participar e assinaram o TCLE, e responderam, nesta sequência aos instrumentos: Questionário Sociodemográfica, Critério Brasil, Inventário de
Habilidades Sociais para Cuidadores de Idosos e Escala de Desejabilidade Social de MarloweCrowne. As coletas foram realizadas de forma individual, duraram em torno de meia hora, e os cuidadores podiam optar por ler e responder ao instrumento sozinhos ou solicitar ajuda da pesquisadora para a leitura das questões em voz alta.

\section{Análise de dados}

Inicialmente foi verificada a normalidade da amostra por meio do teste de normalidade de Kolmogorov-Smirnov (Marôco, 2014). Como a amostra apresentou uma distribuição normal, foi realizado o teste de correlação de Pearson para verificar a existência de correlações entre as medidas desejabilidade social $\mathrm{e}$ as de habilidades sociais dos participantes. Por fim, para avaliar a precisão do IHS-CI foi utilizado o ômega de McDonald. Todas as análises foram realizadas no programa Mplus 7 (Muthén \& Muthén, 2015).

\section{Resultados}

\section{Evidências de validade discriminativa do IHS- CI}

Por meio do coeficiente de correlação de Pearson, foi calculado o índice das correlações entre $\mathrm{o}$ escore de habilidades sociais para cuidadores de idosos (IHS-CI) e o escore de desejabilidade social. Como pode-se observar no Quadro 1, nenhuma medida do IHS-CI apresentou correlação estatisticamente significativa com o instrumento de desejabilidade social. Todas as correlações testadas são apresentadas no Quadro 1.

\section{Precisão}

No presente estudo, o Fator Expressividade Afetiva apresentou $\omega=.85$, o Fator Comunicação Assertiva $\omega=.77$ e 0 Fator Busca de Formação/Informação $\omega=.68$. Todos são considerados valores de precisão adequados (Ventura-León \& Caycho-Rodríguez, 2017). O valor de precisão geral $(\omega=.90)$ apresentou valor considerado excelente. 
Quadro 1. Correlações entre o IHS-CI com a presença de desejabilidade social nos cuidadores

\begin{tabular}{|c|c|c|c|c|}
\hline & \multicolumn{4}{|c|}{ IHS-CI } \\
\hline & Escore Total & $\begin{array}{c}\text { Expressividade } \\
\text { Afetiva }\end{array}$ & $\begin{array}{l}\text { Comunicação } \\
\text { Assertiva }\end{array}$ & $\begin{array}{l}\text { Busca de Formação/ } \\
\text { Informação }\end{array}$ \\
\hline $\begin{array}{l}\text { Escala de Desejabilidade Social } \\
\text { de Marlowe-Crowne }\end{array}$ & $\begin{array}{l}r=0.07 \\
p=.52\end{array}$ & $\begin{array}{l}r=0.14 \\
p=.22\end{array}$ & $\begin{array}{l}r=0.02 \\
p=.89\end{array}$ & $\begin{array}{l}r=-0,03 \\
p=.83\end{array}$ \\
\hline
\end{tabular}

\section{Discussão}

O objetivo deste estudo foi verificar as evidências de validade discriminativa em relação à desejabilidade social e a precisão do IHS-CI por meio do ômega de McDonald. A partir dos resultados encontrados, percebe-se que as hipóteses foram confirmadas. Dessa forma, nenhum fator, nem o escore geral do Inventário de Habilidades Sociais para Cuidadores de Idosos familiares apresentou correlação estatisticamente significativa com a Escala de Desejabilidade Social de Marlowe-Crowne. A falta de correlação indica que o instrumento apresenta evidências de validade discriminativa (AERA, APA, \& NMCE, 2014). Mesmo sendo um instrumento que mede as habilidades sociais, as quais muitas vezes pode levar o participante a dispor de respostas socialmente desejáveis, é possível, a partir destes resultados, afirmar que os construtos são distintos, e o que IHS-CI tem pouca influência do viés de resposta de desejabilidade social (Almiro, 2017). Algo esperado na construção de instrumentos para aferir medidas nas ciências humanas e especificamente na Psicologia (Borsa \& Seize, 2017).

No que tange às medidas de precisão, todas foram adequadas dentre os valores esperados para um instrumento, sendo que o valor de precisão do fator de Expressividade Afetiva e do Escore Geral foram considerados excelentes (Ventura-León \& Caycho-Rodríguez, 2017). Ao se comparar com os valores de estudos anteriores no qual a precisão foi medida pelo Alpha de Cronbach, percebe-se que os valores foram bem similares. Para o fator Expressividade Afetiva, os valores foram $\alpha=.87$ (Queluz et al., 2017), $\alpha=85$ (Queluz et al., 2020) e $\omega=.85$ neste estudo. Já para o fator Comunicação Assertiva, os valores foram $\alpha=.79$ (Queluz et al., 2017), $\alpha=.73$ (Queluz et al., 2020) e $\omega=.77$ neste estudo. No fator Busca de Formação/Informação os valores foram $\alpha=.60$ (Queluz et al., 2017), $\alpha=.68$ (Queluz et al., 2020) e $\omega=.68$ neste estudo. Por fim, os valores do Escore Geral foram $\alpha=.89$
(Queluz et al., 2017), $\alpha=.89$ (Queluz et al., 2020) e $\omega=.90$ neste estudo.

Ao comparar os valores de precisão nos três diferentes estudos do IHS-CI, percebe-se que todos estão dentro dos valores aceitáveis, e que não houve grande diferença ao utilizar o tanto o método de Alpha de Cronbach, como o ômega de McDonald. Uma justificativa para isto é que talvez o número de participantes ou de itens presentes no instrumento seja um número suficiente para garantir um bom valor de precisão, ao se considerar sua influência para o cálculo do Alpha de Cronbach (Ventura-León \& CaychoRodríguez, 2017). Se os estudos iniciais tivessem sido feitos com uma amostra menor, talvez a diferença entre as diferentes formas de mensuração fosse maior. Em estudos futuros, poderá ser interessante comparar os diferentes tipos de medidas de precisão aplicados em amostras de tamanhos diferentes.

Ademais, considerando os objetivos deste estudo e os estudos anteriores realizados por Queluz et al. (2017; 2018; 2020), percebe-se que o IHS-CI está apto para utilização, pois apresenta diferentes tipos de evidências de validade, assim como bons índices de precisão. A partir destas considerações, um próximo passo seria realizar coletas de dados em diferentes regiões do Brasil para verificar se a estrutura encontrada por Queluz et al. (2017) se confirmaria em nível nacional. Em caso positivo, poderia ser viável elaborar um manual de aplicação, estabelecendo uma forma padronizada de aplicar o instrumento a partir de normas pré-estabelecidas e que considerem possíveis diferenças, como por exemplo, em relação ao sexo, escolaridade, região onde mora, entre outros (Borsa \& Seize, 2017).

O IHS-CI apresenta relevância científica e social, uma vez que poderá contribuir para pesquisas que envolvam famílias que estão desenvolvendo o papel de cuidar de um idoso dependente. Futuramente, após a elaboração de seu manual de aplicação, este instrumento poderá ser usado no contexto clínico para intervenções 
tanto individuais como em grupo, visando identificar os déficits e as habilidades sociais mais desenvolvidas em cuidadores de idosos (Queluz et al., 2020). Conforme anteriormente exposto, trabalhar as habilidades sociais nesta população pode contribuir para a melhora no vínculo familiar e também a uma maior disponibilidade de apoio para quem exerce o papel de cuidador principal, levando a uma melhor qualidade de vida de toda a família (Pinto et al., 2016; Ximenes, Queluz, \& Barham, 2019).

Por fim, as limitações deste estudo envolveram uma quantidade reduzida de cuidadores do sexo masculino. No entanto, a quantidade de mulheres tem sido predominante na amostra de cuidadores, uma vez que a tarefa de cuidar é ainda culturalmente atribuída a pessoas do sexo feminino (Ferreira, Isaac, \& Ximenes, 2018; Keating et al., 2019). Em estudos futuros seria importante que mais cuidadores do sexo masculino participassem, a fim de que comparações entre os sexos fossem possíveis de serem realizadas. Além disto, como já dito anteriormente, seria interessante ter uma amostra de cada região do Brasil para que o instrumento fosse representativo e para que fosse possível realizar a comparação entre as diferentes regiões. A partir do exposto, conclui-se que o IHS-CI apresenta boas evidências de validade e precisão, estando apto para uso.

\section{Referências}

Associação Brasileira de Empresas de Pesquisa ABEP (2016). Critério de Classificação Econômica Brasil.

Almiro, P. A. (2017). Uma nota sobre a desejabilidade social e o enviesamento de respostas. Avaliação Psicológica, 16(3), 1. doi:10.15689/ap.2017.1603.ed

American Educational Research Association, American Psychological Association, \& National Council on Measurement in Education (AERA, APA, \& NMCE) (2014). Standards for educational and psychological testing. Washington, DC: American Educational Research Association. doi:10.1002/9780470373699.speced 1992

Beck, A. T., Rush, A. J., Shaw, B. F., \& Emery, G. (1979). Cognitive Therapy of Depression:
A treatment manual. New York: Guilford Press.

Borsa, J. C., \& Seize, M. M. (2017). Construção e adaptação de instrumentos psicológicos: Dois caminhos possíveis. In: B. F. Damásio \& J. C. Borsa (Orgs.). Manual de desenvolvimento de instrumentos psicológicos (pp. 15-37). São Paulo, SP: Vetor.

Crowne, D., \& Marlowe, D. (1960). A new scale of social desirability independent of psychopathology. Journal of Consulting Psychology, 24, 349-354. doi:10.1037/h0047358

Del Prette, Z. A., \& Del Prette, A. (2019). Studies on social skills and social competence in Brazil: A History in Construction. In Koller S. (Ed.), Psychology in Brazil (pp. 41-66). Cham: Springer International Publishing.

Ferreira, C. R., Isaac, L., \& Ximenes, V. S. (2018). Cuidar de idosos: Um assunto de mulher? Estudos Interdisciplinares em Psicologia, 9(1), 108-125. doi:10.5433/2236-6407.2018v9n1p108

Fu, Y. (2019). Care Needs. In D. Gu \& M. E. Dupre (Eds.), Encyclopedia of Gerontology and Population Aging (pp. 1-8). Cham: Springer International Publishing.

Gouveia, V. V., Guerra, V. M., Sousa, D. M. F., Santos, W. S., \& Costa, J. M. (2009). Escala de Desejabilidade Social de MarloweCrowne: Evidências de sua validade fatorial e consistência interna. Avaliação Psicológica, 8(1), 87-98.

Keating, N., Eales, J., Funk, L., Fast, J., \& Min, J. (2019). Life course trajectories of family care. International Journal of Care and Caring, 3(2), 147-63 doi:32/239788219X15473079319309

Marlowe, D., \& Crowne, D. P. (1961). Social desirability and response to perceived situational demands. Journal of Consulting Psychology, 25, 109-115

Marôco, J. (2014). Análise estatística com o SPSS statistics. Pêro Pinheiro, Portugal: Report Number.

Morán, V. E., García, F. E., \& Hormazabal, P. (2018). Validación Transcultural del Cuestionario de Habilidades Sociales para Universitarios en Estudiantes Chilenos y Argentinos (CHS-U). Revista Iberoamericana 
de Diagnóstico y Evaluación - e Avaliação Psicológica, 47(2), 111-123. doi:10.21865/RIDEP47.2.08

Muthén, L.K., \& Muthén, B.O. (2015). Mplus User's Guide. Seventh Edition. Los Angeles, CA: Muthén \& Muthén.

Novelli, M. M. P. C. (2006). Validação da escala de qualidade de vida (QdV-DA) para pacientes com doença de Alzheimer e seus respectivos cuidadores familiares (Tese de doutorado, Programa de Pós Graduação em Medicina, Universidade de São Paulo, SP, Brasil).

Pinto, F. N. F. R., Barham, E. J., \& Del Prette, Z. A. P. (2016). Interpersonal conflicts among family caregivers of the elderly: The importance of social skills. Paidéia (Ribeirão Preto), 26(64), 161-170. doi:10.1590/1982-43272664201605

Queluz, F. N. F. R., Barham, E. J., Del Prette, Z. A. P., Fontaine, A. M. G.V., \& Olaz, F. O. (2017). Inventário de Habilidades Sociais para Cuidadores de Idosos (IHS-CI): Evidências de validade. Avaliação Psicológica, 16(1), 78-86. doi:10.15689/ap.2017.1601.09

Queluz, F. N. F. R., Barham, E. J., Prette, Z. A. P. D., \& Santos, A. A. A. (2018). Inventário de Habilidades Sociais para Cuidadores Familiares de Idosos (IHS-CI): Relações com Indicadores de Bem-Estar Psicológico. Trends in Psychology, 26(2), 537-564. doi:10.9788/tp2018.2-01pt

Queluz, F. N. F. R., Barham, E. J., \& Del Prette, Z. A. P. (2019). The relationship between social skills and psychosocial adjustment among those who care for older adults. Paidéia (RibeirãoPreto), 29, e2917. doi:10.1590/1982-4327e2917

Queluz, F. N. F. R., Santos, A. A. A., Ximenes, V. S., Batista, H. B., \& Kircher, L. F. (2020). Novos estudos psicométricos para o Inventário de Habilidades Sociais para Cuidadores de Idosos (IHS-CI). Relatório Técnico.

Salas, P., Asún, R., \& Zúñiga, C. (2020). Construcción de un Cuestionario de Habilidades Sociales para el Contexto Académico (CHSA). Revista Iberoamericana de Diagnóstico y Evaluación - e Avaliação Psicológica, 55(2), 89-105.
doi:10.21865/RIDEP55.2.07

Scazufca, M. (2002). Versão brasileira da escala Burden Interview para avaliação de sobrecarga em cuidadores de indivíduos com doenças mentais. Revista Brasileira de Psiquiatria, 24(1), 12-17.

United Nations (2019). World Population Ageing 2019 Highlights. New York: United Nations.

Ventura-León, J. L., \& Caycho-Rodríguez, T. (2017). El coeficiente Omega: Un método alternativo para la estimación de la confiabilidad. Revista Latinoamericana de Ciencias Sociales, Niñez y Juventud, 15(1), 625-627.

Vlachantoni, A., \& Palmer, M. F. (2019). Gender and Caring in Later Life. In D. Gu \& M. E. Dupre (Eds.), Encyclopedia of Gerontology and Population Aging (pp. 1-5). Cham: Springer International Publishing.

Wank, X. R., Robinson, K. M, \& Carter-Harris, L. (2014). Prevalence of chronic illnesses and characteristics of chronically ill informal caregivers of persons with dementia. Age and Ageing, 43(1), 137-141.

Ximenes, V. S., Queluz, F. N. F. R., \& Barham, E. J. (2019). Revisão sistemática sobre fatores associados à relação entre habilidades sociais e suporte social. Psico, 50(3), e-31349. doi:10.15448/1980-8623.2019.3.31349

Zarit, S. H., \& Polenick, C. A. (2019). Caregivers' Outcomes. In D. Gu \& M. E. Dupre (Eds.), Encyclopedia of Gerontology and Population Aging (pp. 1-11). Cham: Springer International Publishing. 